OPEN ACCESS

Edited by:

Babajan Banganapalli,

King Abdulaziz University,

Saudi Arabia

Reviewed by:

Munis Dundar,

Erciyes University, Turkey

Richard G. Boles,

Center for Neurological and Neurodevelopmental Health,

United States

*Correspondence:

Maria Rita Passos-Bueno passos@ib.usp.br

${ }^{\dagger}$ Co-first authors

Specialty section:

This article was submitted to

Genetic Disorders,

a section of the journal

Frontiers in Genetics

Received: 15 February 2018 Accepted: 10 April 2018

Published: 25 April 2018

Citation:

Hsia GSP, Musso CM, Alvizi L, Brito LA, Kobayashi GS, Pavanello RCM, Zatz M, Gardham A,

Wakeling E, Zechi-Ceide RM,

Bertola D and Passos-Bueno MR

(2018) Complexity of the 5

Untranslated Region of EIF4A3,

a Critical Factor for Craniofacial

and Neural Development.

Front. Genet. 9:149.

doi: 10.3389/fgene.2018.00149

\section{Complexity of the $5^{\prime}$ Untranslated Region of EIF4A3, a Critical Factor for Craniofacial and Neural Development}

\author{
Gabriella S. P. Hsia't, Camila M. Musso't, Lucas Alvizi', Luciano A. Brito', \\ Gerson S. Kobayashi', Rita C. M. Pavanello', Mayana Zatz'1, Alice Gardham², \\ Emma Wakeling ${ }^{2}$, Roseli M. Zechi-Ceide ${ }^{3}$, Debora Bertola ${ }^{1,4}$ and \\ Maria Rita Passos-Bueno ${ }^{1 *}$
}

' Centro de Estudos do Genoma Humano e Células Tronco, Departamento de Genética e Biologia Evolutiva, Instituto de Biociências, Universidade de São Paulo, São Paulo, Brazil, ${ }^{2}$ North East Thames Genetics Service, Great Ormond Street Hospital, London, United Kingdom, ${ }^{3}$ Hospital de Reabilitação de Anomalias Craniofaciais, Universidade de São Paulo, São Paulo, Brazil, ${ }^{4}$ Instituto da Criança, Hospital das Clínicas da FMUSP, Universidade de São Paulo, São Paulo, Brazil

Repeats in coding and non-coding regions have increasingly been associated with many human genetic disorders, such as Richieri-Costa-Pereira syndrome (RCPS). RCPS, mostly characterized by midline cleft mandible, Robin sequence and limb defects, is an autosomal-recessive acrofacial dysostosis mainly reported in Brazilian patients. This disorder is caused by decreased levels of EIF4A3, mostly due to an increased number of repeats at the EIF4A3 $5^{\prime}$ UTR. EIF4A3 $5^{\prime}$ UTR alleles are CG-rich and vary in size and organization of three types of motifs. An exclusive allelic pattern was identified among affected individuals, in which the CGCA-motif is the most prevalent, herein referred as "disease-associated CGCA-20nt motif." The origin of the pathogenic alleles containing the disease-associated motif, as well as the functional effects of the 5'UTR motifs on EIF4A3 expression, to date, are entirely unknown. Here, we characterized 43 different EIF4A3 5'UTR alleles in a cohort of 380 unaffected individuals. We identified eight heterozygous unaffected individuals harboring the disease-associated CGCA-20nt motif and our haplotype analyses indicate that there are more than one haplotype associated with RCPS. The combined analysis of number, motif organization and haplotypic diversity, as well as the observation of two apparently distinct haplotypes associated with the disease-associated CGCA-20nt motif, suggest that the RCPS alleles might have arisen from independent unequal crossing-over events between ancient alleles at least twice. Moreover, we have shown that the number and sequence of motifs in the $5^{\prime}$ UTR region is associated with EIF4A3 repression, which is not mediated by CpG methylation. In conclusion, this study has shown that the large number of repeats in EIF4A3 does not represent a dynamic mutation and RCPS can arise in any population harboring alleles with the CGCA-20nt motif. We also provided further evidence that EIF4A3 $5^{\prime} U T R$ is a regulatory region and the size and sequence type of the repeats at $5^{\prime}$ UTR may contribute to clinical variability in RCPS.

Keywords: acrofacial dysostosis, non-coding region, haplotype, expansion, crossing-over 


\section{INTRODUCTION}

Over two-thirds of the human genome is comprised by repetitive elements (de Koning et al., 2011), which have been increasingly associated with functional regulatory roles. Consequently, a variety of human genetic disorders are caused by repeats in coding and non-coding sequences (Cummings and Zoghbi, 2000; Gatchel and Zoghbi, 2005; Mirkin, 2007; La Spada and Taylor, 2010; McMurray, 2010; DeJesus-Hernandez et al., 2011; Renton et al., 2011; Usdin et al., 2015; Haeusler et al., 2016). Most of these diseases are caused by unstable dynamic mutations that usually increase in size during meiotic divisions and have been associated with neurologic disorders (Virtaneva et al., 1997; Cummings and Zoghbi, 2000; Renton et al., 2011; Haeusler et al., 2016). However, poly-A repeats in HOXD13, the causative mechanism of a nonneurological condition, synpolydactyly, represents an exception, in which the most likely mechanism leading to increased polyA tracts are errors in DNA replication (Muragaki et al., 1996; Warren, 1997; Brown and Brown, 2004).

We have shown that an increased number of repeats at $5^{\prime} \mathrm{UTR}$ of EIF4A3 causes Richieri-Costa-Pereira syndrome (RCPS; OMIM \#268305), a rare autosomal-recessive disorder affecting craniofacial and limb development, mainly described in Brazilian patients (Favaro et al., 2011, 2014; Bertola et al., 2017). RCPS individuals show a distinctive allelic pattern, determined not only by the larger number of repeats ( $>14$ as compared to up to 12 repeats in controls), but also by the presence of a unique motif containing $G$ instead of $A$ nucleotide (the 'disease-associated CGCA-20nt motif') (Favaro et al., 2014). As the origin of the RCPS disease alleles remains unknown, characterizing the $5^{\prime} \mathrm{UTR}$ of EIF4A3 in a populational level could give us clues on the mechanisms that originate the EIF4A3 pathogenic alleles (e.g., meiotic instability or unequal crossing-over events), in addition to providing insights on the chance of RCPS arising in other populations.

We and others have shown that EIF4A3 downregulation in cellular and animal models leads to defective neural crest cell migration/differentiation and neural stem cell apoptosis during embryonic development, paralleling RCPS cranioskeletal defects and microcephaly, respectively (Mao et al., 2016; Miller et al., 2017). However, the molecular mechanism responsible for EIF4A3 downregulation remains entirely unknown.

Therefore, this work was undertaken to investigate the origin of the pathogenic alleles containing the disease-associated CGCA-20nt motif, as well as to evaluate the functional effects of the $5^{\prime}$ UTR motifs on EIF4A3 expression. Insights into the origin and effect of these complex alleles will contribute to a better understanding of regulatory features of $5^{\prime}$ UTR regions and their role in craniofacial and neural development.

\section{MATERIALS AND METHODS}

\section{Ethics Approval Statement}

The protocol was approved by the Ethics Committee of Instituto de Biociências at Universidade de São Paulo, Brazil (accession number 1.463.852). All individuals donated biological samples after providing signed informed consent.

\section{DNA Samples}

To characterize EIF4A3 5'UTR, 380 DNA samples from unaffected individuals unrelated to RCPS families were selected from the biorepository of CEGH-CEL. For haplotype analysis, 13 additional samples were used, 12 are also from CEHG-CEL (four unaffected individuals without CGCA-20nt motifs and four with CGCA-20nt motifs; four Brazilian RCPS patients bearing different allelic structures) and one sample of a RCPS patient, from the United Kingdom, was sent for diagnosis purposes from North East Thames Genetics Service.

In order to evaluate the effects of the motifs on EIF4A3 expression, six DNA samples carrying EIF4A3 alleles with distinct number of motifs were selected from the biorepository of Centro de Estudos do Genoma Humano e Células Tronco (CEGH-CEL) and used for luciferase reporter assay.

For methylation assessment, we used DNA samples of RCPS patients ( $n=6$; homozygous for the 16 repeats allele) from Hospital de Reabilitação de Anomalias Craniofaciais da Universidade de São Paulo (HRAC-USP) and unaffected individuals ( $n=7$; homozygous for the eight repeats allele) from CEGH-CEL.

All samples were extracted from peripheral blood using the Gentra Systems Autopure LS (AutoGen) according to the manufacturer's protocol.

\section{EIF4A3 5'UTR Characterization and Haplotype Analysis}

Sanger sequencing of EIF4A3 5'UTR (NM_014740.3) and five flanking SNPs (rs11150824, rs2289534, rs3829612, rs10782008, and rs12943620) were performed using BigDye ${ }^{\circledR}$ Terminator v3.1 Cycle Sequencing Kit (Thermo Fisher Scientific) and the ABI 3730 DNA Analyzer (Applied Biosystems). Sequences were analyzed using Sequencher 5.1 (Gene Codes Corporation) and Mixed Sequences Reader (Chang et al., 2012) software. The last one allowed us to better discriminate the alleles. In order to validate our analysis and obtain more reliability in our data, we randomly selected 30 heterozygous samples with different alleles' structure to sequence each allele separately, using the Illustra GFX PCR DNA and Gel Band Purification kit (GE Healthcare) followed by Sanger sequencing.

To facilitate results interpretation, we referred SNPs markers according to their genomic relative position on the annotated plus strand (GRCh38/hg38): SNP1 (rs11150824) SNP2 (rs2289534) - EIF4A3 - SNP3 (rs3829612) - SNP4 (rs10782008) - SNP5 (rs12943620). Primers were designed using Primer-Blast $^{1}$ (Untergasser et al., 2012) and are described in Supplementary Table S1. Linkage disequilibrium and haplotypes were inferred using Haploview software (Barrett et al., 2005).

\section{Luciferase Assay}

Although the larger alleles (15 or 16 repeats) have been associated with decreased EIF4A3 expression, the causal

\footnotetext{
${ }^{1}$ https://www.ncbi.nlm.nih.gov/tools/primer-blast/
} 
relationship between number and/or pattern of repeats and gene downregulation is still unknown. Therefore, we investigated the role of the $5^{\prime}$ UTR motifs on EIF4A3 expression by luciferase assay.

Sequences of interest of unaffected and RCPS individuals were amplified by PCR (primer sequences in Supplementary Table S1), purified using the Illustra GFX PCR DNA and Gel Band Purification kit (GE Healthcare) and cloned into the pGL4.24[luc2P/minP] vector (Promega), upstream of a minimal promoter and the luc $2 P$ gene. Sanger sequencing confirmed all constructs: pGL4.24 vectors carrying control alleles with 4 repeats (3 CACA-20-nt and 1 CA-18-nt), 7 repeats (4 CACA-20-nt, 1 CA-18-nt, 1 CACA-20-nt, and 1 CA-18nt), 10 repeats (7 CACA-20-nt, 1 CA-18-nt, 1 CACA-20-nt, and 1 CA-18-nt) and 12 repeats (1 CACA-20-nt, 10 CGCA20nt, and 1 CA-18-nt); pGL4.24 vectors carrying pathogenic alleles with 14 repeats (2 CACA-20nt, 10 CGCA-20nt, 1 CACA-20nt, and 1 CA-18nt) and 16 repeats (1 CACA-20nt, 13 CGCA-20nt, 1 CACA-20nt, and 1 CA-18nt). In order to investigate the individual effect of each motif, differing in the composition of central nucleotides, we also constructed vectors carrying sequences with only 1 CA-18-nt, 1 CACA-20-nt, and 1 CGCA-20nt, synthesized by Integrated DNA Technologies (IDT).

Human embryonic kidney (HEK) 293T cells, cultured in high glucose DMEM supplemented with $1 \%$ penicillin/streptomycin and $10 \%$ fetal bovine serum (FBS) (all provided by Life Technologies), were plated in 96-well plates $24 \mathrm{~h}$ prior to transfection $\left(2 \times 10^{4}\right.$ cells/well). Transient transfections were performed in triplicate by using TurboFectin 8.0 (OriGene) according to the manufacturer's instruction. Cells were cotransfected with $180 \mathrm{ng}$ of the pGL4.24 constructs and $20 \mathrm{ng}$ of the pRL-SV40 vector (Promega) containing the Renilla luciferase gene, used as a transfection control. The plasmid pLuc generated from pGL3-control template was used as positive control (Soltys et al., 2013). Fortyeight hours after DNA transfection, luciferase activity was measured with the Dual-Glo ${ }^{\circledR}$ Luciferase Assay System in a GloMax Multi 96-microplate Luminometer (Promega). Firefly luminescence results were normalized by Renilla luminescence and the relative luciferase activity was determined. Statistical analyses were performed with oneway ANOVA and Tukey post hoc test. Significance was set at $p<0.05$.

\section{Methylation Assay}

Indeed, methylated $\mathrm{CpGs}$ are involved in gene repression especially when occurring at promoter $/ 5^{\prime}$ UTR (Feil and Fraga, 2012; Schübeler, 2015). Since EIF4A3 5'UTR motifs are CGrich and the disease CGCA-20nt allele shows increased number of CpGs compared to control alleles, we evaluated DNA hypermethylation as a plausible mechanism behind EIF4A3 downregulation in RCPS patients.

One microgram of genomic DNA from each sample were submitted to bisulfite conversion using EpiTect Bisulfite Conversion Kit (QIAgen). Bisulfite converted DNA was subsequently used for PCR, in which primers were designed with MethPrimer $^{2}$ (Li and Dahiya, 2002) and are shown in Supplementary Table S1. Amplicons were checked by agarose electrophoresis and cloned using the TOPO TA Cloning Kit for sequencing (Thermo Fisher Scientific). Sanger sequencing was carried out for 10 clones per sample using the BigDye ${ }^{\circledR}$ Terminator v3.1 Cycle Sequencing Kit (Thermo Fisher Scientific) and the ABI 3730 DNA Analyzer (Applied Biosystems). Sequencing files were then analyzed for methylation quantification using the online tool BISMA Bisulfite Sequencing DNA Methylation Analysis (Rohde et al., 2010) with lower threshold conversion rate at 95\%, lower threshold sequence identity at 90\%, upper threshold of $\mathrm{N}$-sites at cytosine positions at $20 \%$ and per threshold gaps allowed at $20 \%$ as filtering parameters. Methylation values were computed and differences between groups tested using Fisher's Exact Test. Significance was set at $p<0.05$.

\section{RESULTS}

The EIF4A3 5'UTR is characterized by the presence of 18 - or 20-nucleotide-long motifs differing in the composition of central nucleotides, namely CA-18nt (TCGGCAGCGGCAGCGAGG), CACA-20nt (TCGGCAGCGGCACAGCGAGG), and CGCA20nt (TCGGCAGCGGCGCAGCGAGG) (Favaro et al., 2014). Unaffected individuals have 3-12 repeats composed mostly by CA-18nt and CACA-20nt motifs, while RCPS patients have 14-16 repeats, with a higher number of the CGCA-20nt motif (Favaro et al., 2014). The motif CGCA-20nt will be herein referred as 'disease-associated CGCA-20nt motif.'

\section{Unaffected Individuals Are Mostly Heterozygous and May Also Present CGCA-20nt Motifs}

Sanger sequencing of EIF4A3 $5^{\prime}$ UTR in 380 unaffected individuals revealed 43 different alleles (Figure 1) in heterozygosis in $85 \%$ of individuals. The total number of repeats per allele varied from 2 to 17 , and the most common alleles contained $7(25.46 \%)$ or $8(23.21 \%)$ repeats (Supplementary Figure S1). The number and organization of the CA-18nt or CACA-20nt motifs varied between the alleles. We identified not only alleles containing a single motif, but also complex alleles with multiple organizations of the CA-18nt or CACA-20nt motifs (Figure 1). For example, one allele exclusively constituted by the CA-18nt motif was found. As for the other alleles, the number of the first CACA-20nt motifs varied from 2 to 8 with or without different combinations of CA-18nt motifs (Figure 1). The most common allele contained seven repeats and was constituted by four repeats of CACA-20nt motif followed by 1 CA-18nt, 1 CACA-20nt, and 1 final CA-18nt. We also identified two atypical alleles comprising 12- or 20-nucleotide-long sequences inserted between motifs, which do not align to any viral sequences or neighboring genes (data not shown). Finally, the diseaseassociated CGCA-20nt motif, originally found only among RCPS patients, was identified here in eight heterozygous control

${ }^{2}$ www.urogene.org/methprimer/ 


\begin{tabular}{|c|c|c|}
\hline Allele ID & Allele structure $\left(5^{\prime} \rightarrow 3^{\prime}\right)$ & NR \\
\hline $\mathrm{A} \# 1$ & $\infty$ & 3 \\
\hline $\mathrm{A} \# 2$ & $\infty$ & 2 \\
\hline A\#3 & $\infty 00$ & 3 \\
\hline A\#4 & $\infty 00$ & 4 \\
\hline A\#5 & $\infty 000$ & 5 \\
\hline $\mathrm{A} \# 6$ & $\infty 0000$ & 6 \\
\hline $\mathrm{A} \# 7$ & 0000000 & 7 \\
\hline $\mathrm{A} \# 8$ & 0000000 & 8 \\
\hline $\mathrm{A} \# 9$ & 000 & 4 \\
\hline $\mathrm{A} \# 10$ & 0000 & 5 \\
\hline A\#11 & 00000 & 6 \\
\hline $\mathrm{A \# 12}$ & 0000000 & 7 \\
\hline $\mathrm{A} \# 13$ & 00000000 & 8 \\
\hline $\mathrm{A} \# 14$ & 000000000 & 9 \\
\hline A\#15 & 0000000000 & 10 \\
\hline A\#16 & 0000 & 5 \\
\hline A\#17 & 00000 & 7 \\
\hline $\mathrm{A \# 18}$ & 0000000 & 8 \\
\hline A\#19 & 000000000 & 9 \\
\hline $\mathrm{A} \# 20$ & 000000 & 7 \\
\hline $\mathrm{A} \# 21$ & 0000 & 5 \\
\hline $\mathrm{A} \# 22$ & 00000 & 6 \\
\hline$A \# 23$ & 000000 & 7 \\
\hline A\#24 & 0000000 & 8 \\
\hline $\mathrm{A} \# 25$ & 0000 & 5 \\
\hline $\mathrm{A} \# 26$ & 00000 & 6 \\
\hline $\mathrm{A} \# 27$ & 000000 & 7 \\
\hline A\#28 & 0000000 & 8 \\
\hline $\mathrm{A} \# 29$ & 00000000 & 9 \\
\hline A\#30 & 0000000000 & 10 \\
\hline A\#31 & 00000000000 & 11 \\
\hline A\#32 & 0000000 & 7 \\
\hline$A \# 33$ & 0000000 & 8 \\
\hline A\#34 & 000000 & 7 \\
\hline A\#35 & 00000000 & 8 \\
\hline A\#36 & 00000000 & 9 \\
\hline A\#37 & 0000000 & 8 \\
\hline A\#38 & 0000000 & 8 \\
\hline $\mathrm{A} \# 39$ & 0000 -12-uxcleotide-long-sequence- & 6 \\
\hline $\mathrm{A} \# 40$ & 000000000 -20-mucleodide-long-senunence- & 10 \\
\hline $\mathrm{A} \# 41$ & 00000000000 & 11 \\
\hline $\mathrm{A} \# 42$ & 000000000000 & 12 \\
\hline $\mathrm{A} \# 43$ & 0000000000000000 & 17 \\
\hline
\end{tabular}

CA-18nt CACA-20nt CGCA-20n NR - Number of repeats
FIGURE 1 | Schematic representation of the 43 allele structures identified in the 380 unaffected individuals.

individuals, within alleles with 11 or more repeats (Figure 1). The largest allele (17 repeats) was found in heterozygosis in one control individual and contained 14 CGCA-20nt motifs. This allele must be pathogenic when in homozygosis.

\section{Haplotype Analysis Suggests That the RCPS Allele Originated More Than Once}

To understand the origin of the alleles containing the diseaseassociated CGCA-20nt motif, we initially characterized the haplotypes of 13 samples: five affected individuals (four Brazilian and one from the United Kingdom) and eight control individuals (Supplementary Table S2), using five SNPs flanking EIF4A3 and spanning $519 \mathrm{~kb}$ (Figure 2A). We observed a weak linkage disequilibrium in this block $\left(D^{\prime}<0.48\right)$.
The Brazilian RCPS patients showed at least two different haplotypes. The haplotype associated with the pathogenic 14-repeat allele is different from the one associated with the 16repeat allele (Figure 2B), which in turn were not observed in any of the tested unaffected individuals (Figure 2C). The fact that the two affected alleles with 14 and 16 repeats are embedded within different haplotypes, undetected in unaffected individuals, suggests a distinct origin for these two different-sized pathogenic alleles in our population. Next, we analyzed the homozygous (16 repeats) UK RCPS sample, which showed a different haplotype from those observed in the Brazilian RCPS (Figure 2B). However, one UK RCPS haplotype is similar to a haplotype observed in three of the unaffected individuals carrying the disease-associated CGCA-20nt motif (Figures 2B,D). Besides, the alleles in these control individuals also show similarities in motif organization in relation to the allele in the UK RCPS patient, suggesting that these alleles possibly share a common origin (Figure $2 \mathbf{E}$ and Supplementary Table S2).

\section{Transcriptional Activity Is Inversely Correlated to Motif Number at EIF4A3 $5^{\prime}$ UTR}

To clarify the functional role of the allelic structure at EIF4A3 $5^{\prime}$ UTR, we generated constructs varying in size and composition (Figure 3), and carried out luciferase reporter assays. Inverse correlation between number of motifs and luciferase activity was observed ( $n=4$ independent experiments; $p<0.05$; Figure 3A). Further, by investigating each motif individually, there was a discrete reduction in expression for the disease-associated CGCA-20nt, albeit not statistically significant $(n=3$ independent experiments; Figure 3B).

In order to address whether the motif sequence plays a role in EIF4A3 gene expression, based on data shown in Figure 3A, we calculated the effect per motif type on luciferase activity. Comparing the luciferase activity of alleles with similar composition of motifs, carrying 4 and 10 repeats $(22.45 \%$ and $19.69 \%$, respectively), we observed a decrease of $2.76 \%$ in expression, which represents a reduction of about $0.46 \%$ per CACA-20nt motif added in the allele structure. On the other hand, between the alleles carrying 12 and 16 repeats $(17.8 \%$ and $11.96 \%$, respectively), with comparable allelic structure, the difference was $5.84 \%$, which means a reduction in luciferase activity of $1.46 \%$ per CGCA-20nt motif added (three times higher). Based on these results, we suggest that both size and allele sequence play a role in gene regulation.

\section{5'UTR Hypermethylation Is Not Responsible for EIF4A3 Downregulation}

We inspected methylation levels at the $5^{\prime} \mathrm{UTR}$ of EIF4A3, as the increased number of repeats in RCPS patients leads to gain of 37 CpG sites. We observed that both RCPS and controls did not show abundant methylation of this region $(1.7 \%$ and $2.8 \%$ of methylated CpGs, respectively), with a discrete reduction of methylation in RCPS ( $p<0.05)$ (Supplementary Table S3). There was no evident methylation variation at any specific $\mathrm{CpG}$ 


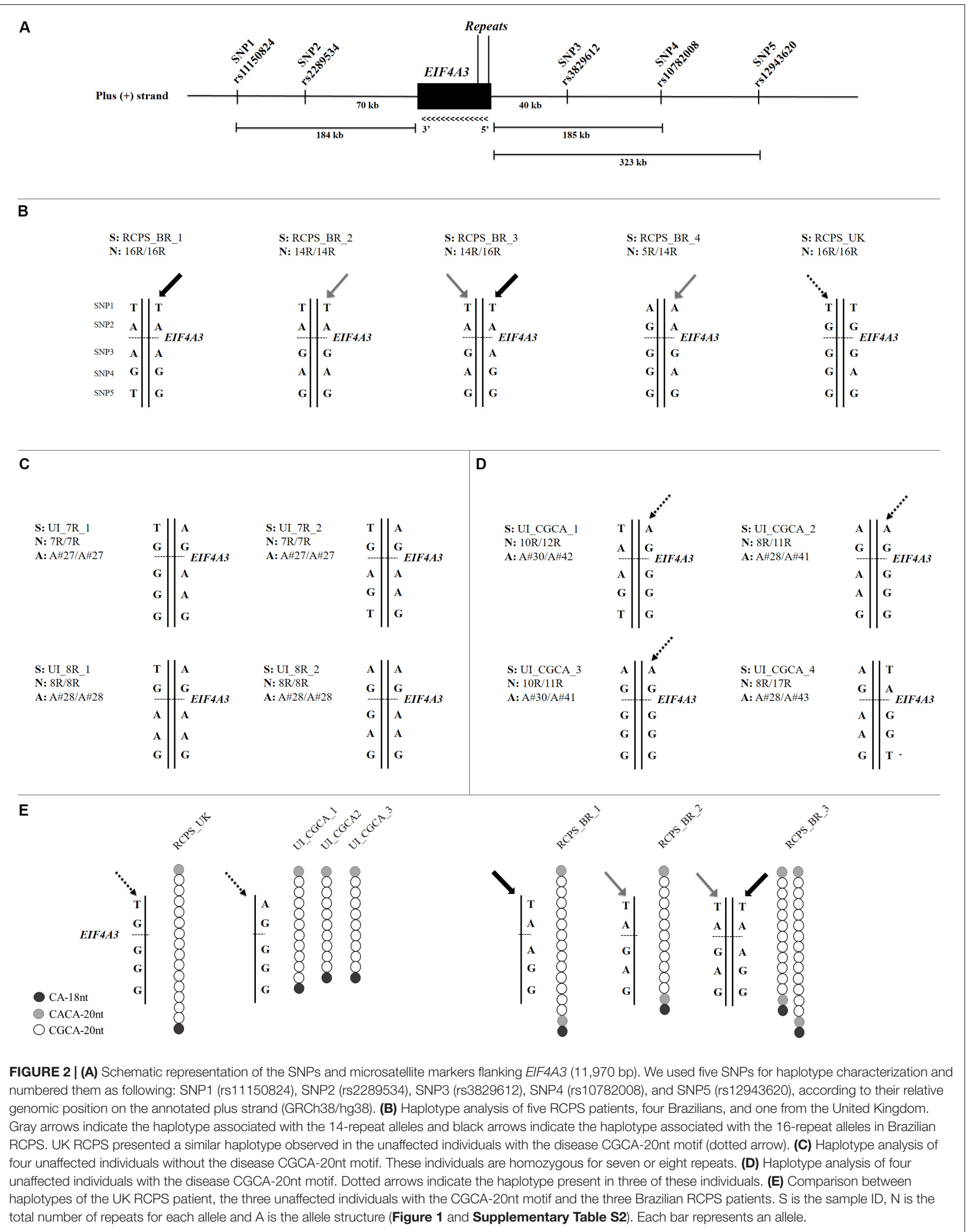




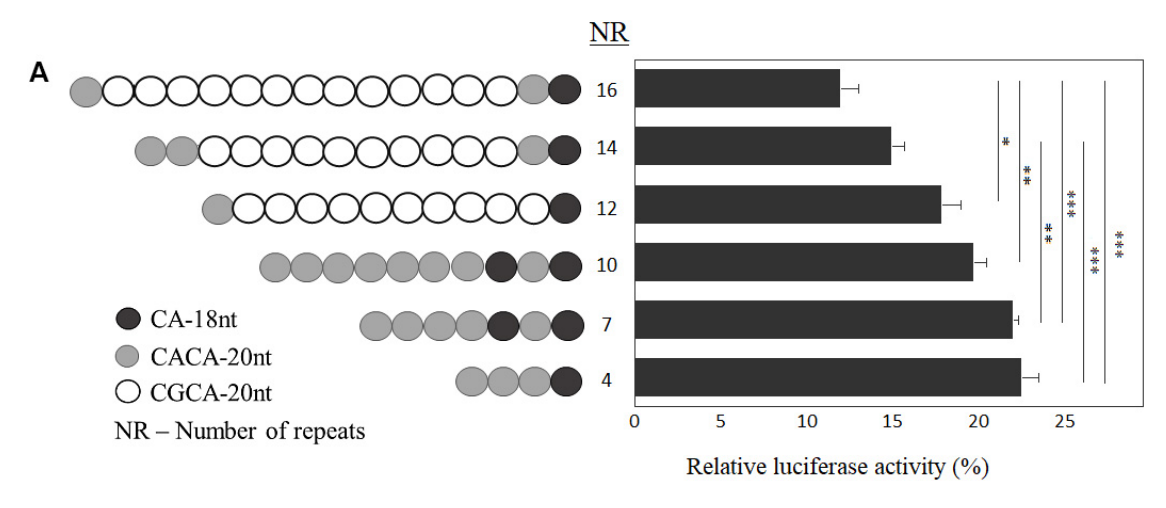

B

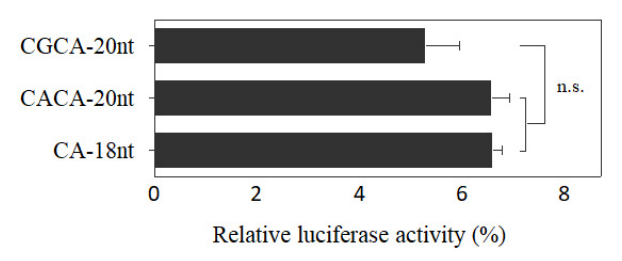

FIGURE 3 | Functional analysis of EIF4A3 5'UTR motifs. Graph depicting the relative luciferase activity, in percentage, of the constructs carrying different number of motifs (A) and of each motif independently (B). Data are represented as mean \pm SEM of five experiments, which were performed in triplicate. Relative luciferase activity $=[($ experimental sample ratio) - (negative control ratio)/(positive control ratio) - (negative control ratio) $] . * * p<0.001, * * p<0.01, * p<0.05$ and $n s$, non-significant; one-way ANOVA with Tukey post hoc tests.

between RCPS and controls, as both presented low methylation levels.

\section{DISCUSSION}

Expansions at non-coding regions have been extensively described in neurological disorders, and characterization of these regions have greatly contributed to the understanding of novel regulatory mechanisms (Gatchel and Zoghbi, 2005; Mirkin, 2007; La Spada and Taylor, 2010; McMurray, 2010; Russo et al., 2015; Usdin et al., 2015; Haeusler et al., 2016). Despite the great advances in genome sequence analysis, DNA of repetitive regions is still difficult to be sequenced. In fact, the EIF4A3 $5^{\prime}$ UTR is not covered in GnomAD database, which reinforces the importance of characterizing this region through Sanger sequencing.

In this study, Sanger sequencing analysis of EIF4A3 $5^{\prime} \mathrm{UTR}$ in 380 unaffected individuals revealed 43 different alleles, with the most common alleles containing seven or eight repeats. Some of these alleles presented only one type of motif (CA18nt or CACA-20nt) with different total number of repeats, while others presented a visible combination of these two common motifs, suggesting that these alleles may have originated through unequal crossing-over events. These results show its polymorphic nature and confirm the structural complexity, and uniqueness of this region, which was not comparable to any gene in which dynamic pathogenic expansions at non-coding regions had been reported (Brook et al., 1992; Campuzano et al., 1996; Moseley et al., 2006; Daughters et al., 2009; Galloway and Nelson, 2009; Sato et al., 2009; DeJesus-Hernandez et al., 2011; Kobayashi et al., 2011). In this enlarged cohort, $1 \%$ of the alleles (8/760) harbors the disease-associated CGCA-20nt motif. Interestingly, the largest alleles in this cohort ( $\geq 11$ repeats), including one with 17 repeats, contained the disease CGCA20nt motif. These results suggest that RCPS could occur in any population containing alleles with the CGCA-20nt motif. Indeed, one of the patients here included is from United Kingdom (Bertola et al., 2017).

The haplotype analyses were performed in order to get insights on the origin of the pathogenic alleles with increased number of repeats. Results revealed that the pathogenic alleles with 14 and 16 repeats of Brazilian patients have distinct origins, which in turn are different from the haplotypes of the UK RCPS patient. These results suggest that the pathogenic alleles have arisen more than once. It is of note that the UK RCPS patient shares a common haplotype and also a similar motif structure with three unaffected individuals carrying the disease-associated CGCA20nt motif, suggesting a common ancestral among them. It is possible that, similarly to the alleles in the control population, the affected alleles may have arisen also through unequal crossing. This hypothesis is also supported by the observation that the number of repeats at EIF4A3 5'UTR seems to be stable across generations (Favaro et al., 2014). This phenomenon is more comparable to the one observed in synpolydactyly, in which the poly-A at the $3^{\prime}$ end of HOXD13 might have originated by unequal crossing over as it is quite stable when transmitted across generations (Warren, 1997), as opposed to dynamic mutations observed in neurological conditions, which have arisen only once (Virtaneva et al., 1997; Gatchel and Zoghbi, 2005; Mirkin, 2007; La Spada and Taylor, 2010; McMurray, 2010; DeJesus-Hernandez et al., 2011; Renton et al., 2011; Usdin et al., 2015; Haeusler et al., 2016). 
Next, we demonstrated that the number and allele sequence of motifs at $5^{\prime} \mathrm{UTR}$ is involved in EIF4A3 expression. These results thus suggest a potential cis-acting regulatory mechanism for these motifs on gene expression and confirm our previous finding that the affected alleles were associated with EIF4A3 downregulation in cells from different tissues from RCPS patients, including peripheral blood (Favaro et al., 2014; Miller et al., 2017). DNA hypermethylation is not the mechanism for EIF4A3 downregulation in RCPS patients, as RCPS and control blood samples showed similar methylation levels. Hypomethylation, as observed at EIF4A3 5'UTR, is consistent with transcriptional activity (Baubec and Schübeler, 2014; Schübeler, 2015). There could be alternative mechanisms by which the increased number of motifs could repress EIF4A3 expression: the disease CGCA-20nt could create binding sites for repressor proteins and act as a silencer; or more complex mechanisms, including post-translational events, could be involved. Further functional studies are needed to pinpoint the exact molecular mechanism underlying EIF4A3 downregulation.

The inverse correlation between number of repeats and EIF4A3 expression levels suggests that the structure of the 5'UTR EIF4A3 may modulate the phenotype. Indeed, a broader phenotypic spectrum within RCPS has been observed, ranging from individuals with severe phenotype (homozygous for the 16-repeat allele or heterozygous for the 15- and 16-repeat alleles) to individuals with less severe skeletal involvements, harboring smaller number of motifs (homozygous for the 14repeat allele or compound heterozygous for 14 repeats and a point mutation (c.809A > G) (Favaro et al., 2014; Bertola et al., 2017). Despite limitations in sample size, these results suggest that RCPS phenotypic variability depends upon the number and allele sequence of repeats at the $5^{\prime}$ UTR of EIF4A3.

\section{CONCLUSION}

In summary, we provide evidence supporting that the EIF4A3 5'UTR is highly polymorphic, comprising at least 43 different alleles, which may have originated through extensive recombination in this region. Haplotype analysis in control and in affected individuals suggests that there are more than one haplotype associated with the disease and RCPS alleles might have originated from unequal crossing-over events. We also provided further evidence that EIF4A3 $5^{\prime} \mathrm{UTR}$ is a regulatory region, and that EIF4A3 downregulation in RCPS is not mediated by CpG methylation. Moreover, our findings provide insights to explain clinical variability in RCPS.

\section{REFERENCES}

Barrett, J. C., Fry, B., Maller, J., and Daly, M. J. (2005). Haploview: analysis and visualization of LD and haplotype maps. Bioinformatics 21, 263-265. doi: 10. 1093/bioinformatics/bth457

Baubec, T., and Schübeler, D. (2014). Genomic patterns and context specific interpretation of DNA methylation. Curr. Opin. Genet. Dev. 25, 85-92. doi: 10.1016/j.gde.2013.11.015

\section{AUTHOR CONTRIBUTIONS}

DB, RP, and MZ provided the Brazilian DNA samples. AG and EW provided DNA sample and clinical information from the UK RCPS patient. GH, CM, and MP-B conceived and designed the study. GH performed the EIF4A3 5'UTR characterization and haplotype analyses. LB assisted with haplotype and linkage disequilibrium analyses. CM carried out the luciferase assay and LA performed the methylation assay. GH, CM, LA, LB, GK, and MP-B discussed and interpreted the results and wrote the manuscript. $\mathrm{GH}$ and $\mathrm{CM}$ have a major contribution in writing the manuscript. All authors contributed to the final version and approved the manuscript.

\section{FUNDING}

This work was funded by Centro de Pesquisa, Inovação e Difusão (CEPID, 2013/08028-1), Fundação de Amparo à Pesquisa do Estado de São Paulo (FAPESP, 2015/21781-6), Conselho Nacional de Desenvolvimento Científico e Tecnológico (CNPq, PQ10/2011), and Coordenação de Aperfeiçoamento de Pessoal de Nível Superior (CAPES, 1470032).

\section{ACKNOWLEDGMENTS}

We thank Dr. Meire Aguena and Dr. Antonia Cerqueira (IBUSP) for technical support. We also thank Dr. Marcelo Nobrega and Dr. Débora Sobreira (University of Chicago, United States) who donated the sequences for constructs used in the luciferase assay.

\section{SUPPLEMENTARY MATERIAL}

The Supplementary Material for this article can be found online at: https://www.frontiersin.org/articles/10.3389/fgene. 2018.00149/full\#supplementary-material

FIGURE S1 | Distribution of number of alleles per total number of repeats identified in the 380 samples of unaffected individuals. This analysis did not take in consideration the motifs' structure.

TABLE S1 | Primer sequences.

TABLE S2 | List of the 13 samples used in haplotype analysis and the description of motifs' structure reported in each allele.

TABLE S3 | Methylation levels in the analyzed regions in RCPS patients and control individuals; $p=0.0461$, Fisher's Exact Test.

Bertola, D. R., Hsia, G., Alvizi, L., Gardham, A., Wakeling, E. L., Yamamoto, G. L., et al. (2017). Richieri-Costa-Pereira syndrome: expanding its phenotypic and genotypic spectrum. Clin. Genet. 93, 800-811. doi: 10.1111/cge. 13169

Brook, J. D., McCurrach, M. E., Harley, H. G., Buckler, A. J., Church, D., Aburatani, H., et al. (1992). Molecular basis of myotonic dystrophy: expansion of a trinucleotide (CTG) repeat at the $3^{\prime}$ end of a transcript encoding a protein kinase family member. Cell 68, 799-808. doi: 10.1016/0092-8674(92)90154-5 
Brown, L. Y., and Brown, S. A. (2004). Alanine tracts: the expanding story of human illness and trinucleotide repeats. Trends Genet. 20, 51-58. doi: 10.1016/j.tig. 2003.11.002

Campuzano, V., Montermini, L., Moltò, M. D., Pianese, L., Cossée, M., Cavalcanti, F., et al. (1996). Friedreich's ataxia: autosomal recessive disease caused by an intronic GAA triplet repeat expansion. Science 271, 1423-1427. doi: 10.1126/science.271.5254.1423

Chang, C.-T., Tsai, C.-N., Tang, C. Y., Chen, C.-H., Lian, J.-H., Hu, C.-Y., et al. (2012). Mixed sequence reader: a program for analyzing DNA sequences with heterozygous base calling. ScientificWorldJournal 2012:365104. doi: 10.1100/ $2012 / 365104$

Cummings, C. J., and Zoghbi, H. Y. (2000). Fourteen and counting: unraveling trinucleotide repeat diseases. Hum. Mol. Genet. 9, 909-916. doi: 10.1093/hmg/ 9.6.909

Daughters, R. S., Tuttle, D. L., Gao, W., Ikeda, Y., Moseley, M. L., Ebner, T. J., et al. (2009). RNA gain-of-function in spinocerebellar ataxia type 8. PLoS Genet. 5:e1000600. doi: 10.1371/journal.pgen.1000600

de Koning, A. P., Gu, W., Castoe, T. A., Batzer, M. A., and Pollock, D. D. (2011). Repetitive elements may comprise over two-thirds of the human genome. PLoS Genet. 7:e1002384. doi: 10.1371/journal.pgen.1002384

DeJesus-Hernandez, M., Mackenzie, I. R., Boeve, B. F., Boxer, A. L., Baker, M., Rutherford, N. J., et al. (2011). Expanded GGGGCC hexanucleotide repeat in noncoding region of C9ORF72 causes chromosome 9p-linked FTD and ALS. Neuron 72, 245-256. doi: 10.1016/j.neuron.2011.09.011

Favaro, F. P., Alvizi, L., Zechi-Ceide, R. M., Bertola, D., Felix, T. M., de Souza, J., et al. (2014). A noncoding expansion in EIF4A3 causes Richieri-Costa-Pereira syndrome, a craniofacial disorder associated with limb defects. Am. J. Hum. Genet. 94, 120-128. doi: 10.1016/j.ajhg.2013.11.020

Favaro, F. P., Zechi-Ceide, R. M., Alvarez, C. W., Maximino, L. P., Antunes, L. F., Richieri-Costa, A., et al. (2011). Richieri-Costa-Pereira syndrome: a unique acrofacial dysostosis type. An overview of the Brazilian cases. Am. J. Med. Genet. A 155A, 322-331. doi: 10.1002/ajmg.a.33806

Feil, R., and Fraga, M. F. (2012). Epigenetics and the environment: emerging patterns and implications. Nat. Rev. Genet. 13, 97-109. doi: 10.1038/nrg3142

Galloway, J. N., and Nelson, D. L. (2009). Evidence for RNA-mediated toxicity in the fragile X-associated tremor/ataxia syndrome. Future Neurol. 4:785. doi: $10.2217 /$ fnl.09.44

Gatchel, J. R., and Zoghbi, H. Y. (2005). Diseases of unstable repeat expansion: mechanisms and common principles. Nat. Rev. Genet. 6, 743-755. doi: 10.1038/ nrg1691

Haeusler, A. R., Donnelly, C. J., and Rothstein, J. D. (2016). The expanding biology of the C9orf72 nucleotide repeat expansion in neurodegenerative disease. Nat. Rev. Neurosci. 17, 383-395. doi: 10.1038/nrn.2016.38

Kobayashi, H., Abe, K., Matsuura, T., Ikeda, Y., Hitomi, T., Akechi, Y., et al. (2011). Expansion of intronic GGCCTG hexanucleotide repeat in NOP56 causes SCA36, a type of spinocerebellar ataxia accompanied by motor neuron involvement. Am. J. Hum. Genet. 89, 121-130. doi: 10.1016/j.ajhg.2011.05.015

La Spada, A. R., and Taylor, J. P. (2010). Repeat expansion disease: progress and puzzles in disease pathogenesis. Nat. Rev. Genet. 11, 247-258. doi: 10.1038/ nrg2748

Li, L.-C., and Dahiya, R. (2002). MethPrimer: designing primers for methylation PCRs. Bioinformatics 18, 1427-1431. doi: 10.1093/bioinformatics/18.11.1427

Mao, H., McMahon, J. J., Tsai, Y. H., Wang, Z., and Silver, D. L. (2016). Haploinsufficiency for core exon junction complex components disrupts embryonic neurogenesis and causes p53-mediated microcephaly. PLoS Genet. 12:e1006282. doi: 10.1371/journal.pgen.1006282

McMurray, C. T. (2010). Mechanisms of trinucleotide repeat instability during human development. Nat. Rev. Genet. 11, 786-799. doi: 10.1038/nrg2828

Miller, E. E., Kobayashi. G. S., Musso, C. M., Allen, M., Ishiy, F. A. A., de Caires, L. C., et al. (2017). EIF4A3 deficient human iPSCs and mouse models demonstrate neural crest defects that underlie Richieri-CostaPereira syndrome. Hum. Mol. Genet. 26, 2177-2191. doi: 10.1093/hmg/ ddx078

Mirkin, S. M. (2007). Expandable DNA repeats and human disease. Nature 447, 932-940. doi: 10.1038/nature05977

Moseley, M. L., Zu, T., Ikeda, Y., Gao, W., Mosemiller, A. K., Daughters, R. S., et al. (2006). Bidirectional expression of CUG and CAG expansion transcripts and intranuclear polyglutamine inclusions in spinocerebellar ataxia type 8. Nat. Genet. 38, 758-769. doi: 10.1038/ng1827

Muragaki, Y., Mundlos, S., Upton, J., and Olsen, B. R. (1996). Altered growth and branching patterns in synpolydactyly caused by mutations in HOXD13. Science 272, 548-551. doi: 10.1126/science.272.5261.548

Renton, A. E., Majounie, E., Waite, A., Simón-Sánchez, J., Rollinson, S., Gibbs, J. R., et al. (2011). A hexanucleotide repeat expansion in C9ORF72 is the cause of chromosome 9p21-linked ALS-FTD. Neuron 72, 257-268. doi: 10.1016/j. neuron.2011.09.010

Rohde, C., Zhang, Y., Reinhardt, R., and Jeltsch, A. (2010). BISMA-fast and accurate bisulfite sequencing data analysis of individual clones from unique and repetitive sequences. BMC Bioinformatics 11:230. doi: 10.1186/1471-210511-230

Russo, A., Pacchierotti, F., Cimini, D., Ganem, N. J., Genescà, A., Natarajan, A. T., et al. (2015). Genomic instability: crossing pathways at the origin of structural and numerical chromosome changes. Environ. Mol. Mutagen. 56, 563-580. doi: 10.1002/em.21945

Sato, N., Amino, T., Kobayashi, K., Asakawa, S., Ishiguro, T., Tsunemi, T., et al. (2009). Spinocerebellar ataxia type 31 is associated with "inserted" pentanucleotide repeats containing (TGGAA)n. Am. J. Hum. Genet. 85, 544-557. doi: 10.1016/j.ajhg.2009.09.019

Schübeler, D. (2015). Function and information content of DNA methylation. Nature 517, 321-326. doi: 10.1038/nature14192

Soltys, D. T., Rocha, C. R., Lerner, L. K., de Souza, T. A., Munford, V., Cabral, F., et al. (2013). Novel XPG (ERCC5) mutations affect DNA repair and cell survival after ultraviolet but not oxidative stress. Hum. Mutat. 34, 481-489. doi: 10.1002/humu.22259

Untergasser, A., Cutcutache, I., Koressaar, T., Ye, J., Faircloth, B. C., Remm, M., et al. (2012). Primer3-new capabilities and interfaces. Nucleic Acids Res. 40:e115. doi: 10.1093/nar/gks596

Usdin, K., House, N. C., and Freudenreich, C. H. (2015). Repeat instability during DNA repair: insights from model systems. Crit. Rev. Biochem. Mol. Biol. 50, 142-167. doi: 10.3109/10409238.2014.999192

Virtaneva, K., D’Amato, E., Miao, J., Koskiniemi, M., Norio, R., Avanzini, G., et al. (1997). Unstable minisatellite expansion causing recessively inherited myoclonus epilepsy, EPM1. Nat. Genet. 15, 393-396. doi: 10.1038/ng0 497-393

Warren, S. T. (1997). Polyalanine expansion in synpolydactyly might result from unequal crossing-over of HOXD13. Science 275, 408-409. doi: 10.1126/science. 275.5298.408

Conflict of Interest Statement: The authors declare that the research was conducted in the absence of any commercial or financial relationships that could be construed as a potential conflict of interest.

Copyright (c) 2018 Hsia, Musso, Alvizi, Brito, Kobayashi, Pavanello, Zatz, Gardham, Wakeling, Zechi-Ceide, Bertola and Passos-Bueno. This is an open-access article distributed under the terms of the Creative Commons Attribution License (CC BY). The use, distribution or reproduction in other forums is permitted, provided the original author(s) and the copyright owner are credited and that the original publication in this journal is cited, in accordance with accepted academic practice. No use, distribution or reproduction is permitted which does not comply with these terms. 